\title{
Dimensiones culturales en el proceso de atención primaria infantil: perspectivas de las madres
}

\author{
Ana María Alarcón-Muñoz, PhD en Antrop. Méd., (1) \\ Aldo Conrado Vidal-Herrera, $M$ en $\mathrm{CS}^{(2)}$
}

\begin{abstract}
Alarcón-Muñoz AM,Vidal-Herrera AC.
Dimensiones culturales en el proceso de atención primaria infantil: perspectivas de las madres. Salud Publica Mex 2005;47:440-446.
\end{abstract}

\section{Resumen}

Objetivo. Explorar las dimensiones culturales en el proceso de atención primaria infantil desde la perspectiva de las madres que acuden a centros de salud primaria en la región de la Araucania, Chile. Material y métodos. Estudio cualitativo realizado durante el año 2003 en la zona de mayor índice de etnicidad del país. Los participantes fueron 94 madres mapuches y no mapuches a quienes se le aplicó una entrevista en profundidad. Resultados. Tres dimensiones culturales surgieron del análisis: a) modelos explicativos de enfermedad asociados a factores culturales, político-económicos, y ambientales; b) itinerario terapéutico que combina recursos de los sistemas indígena, popular y biomédico; y c) falta de competencia cultural del sistema de atención. Conclusiones. Las madres conciben las enfermedades infantiles articulando componentes mágico-religiosos, y naturales (frío, calor, humedad). La atención de salud infantil enfrenta un importante desafío comunicacional en la superación de barreras sociales y lingüísticas entre madres y personal de salud.

Palabras clave: atención primaria infantil; modelos médicos; salud y cultura; pertinencia cultural; itinerario terapéutico; mapuche; Chile
Alarcón-Muñoz AM,Vidal-Herrera AC.

Cultural dimensions of the childhood primary health care delivery from the mothers' perceptions in the Araucania-Chile. Salud Publica Mex 2005;47:440-446.

\section{Abstract}

Objective. To explore the cultural dimensions of the childhood primary health care delivery process from the mothers' perceptions in the Araucania region of Chile. Material and Methods. Qualitative study performed in the year 2003 within the zone with the highest ethnicity rate of the country. Ninety four Mapuche and non-Mapuche mothers agreed to be in depth interviewed. Results. The analysis drew three cultural dimensions: a) Explanatory models of disease were associated with cultural, political-economy, and environmental factors; b) The therapeutic itinerary blends indigenous, popular, and biomedical resources and; c) Health care delivery process lacks of cultural competence. Conclusions. The mothers explain their children diseases articulating religious, magic, and natural (hot, cold, humidity) causes. The main challenge of the primary healthcare delivery process is to overcome the communicational barriers due to the social and linguistic differences between mothers and health care providers.

Key words: childhood primary healthcare; medical models; health and culture; cultural competence; therapeutic itinerary; Mapuche; Chile

(I) Departamento de Salud Pública, Facultad de Medicina, Universidad de La Frontera, Chile.

(2) Departamento de Ciencias Sociales, Facultad de Humanidades, Universidad de La Frontera, Chile.

Fecha de recibido: 26 de octubre de 2004 - Fecha de aprobado: 25 de agosto de 2005 Solicitud de sobretiros: Dra. Ana María Alarcón Muñoz. Departamento de Salud Pública, Facultad de Medicina. Universidad de La Frontera, Casilla 54-D, Temuco, Chile Correo electrónico: amalarc@ufro.cl 
$\mathrm{L}$ a determinación de los problemas de salud de la población es fundamental en el proceso de planificación sanitaria tanto en el plano nacional como en el local. Sin embargo, la manera en que los problemas de salud se perciben puede tener diversas orientaciones. En el nivel macro, por ejemplo, las autoridades de salud suelen preocuparse por problemas sanitarios de largo plazo, como la disminución de la mortalidad, el diseño de políticas, la creación de estructuras para la satisfacción de necesidades sanitarias o la definición de criterios para focalizar los recursos del sector. En el nivel intermedio, el personal de salud percibe la importancia de un problema sanitario desde un punto de vista que refleja su experiencia en el campo clínico, su familiaridad con las estadísticas locales, regionales o nacionales, y la literatura médica. Finalmente, la percepción de los usuarios a menudo no es estrictamente médica, pues para estos la falta de transporte, la ausencia de un seguro de salud, la pobreza, la discriminación social e incluso la calidad de las relaciones humanas entre el personal de salud y los pacientes pueden ser factores determinantes en la percepción de los problemas sanitarios. De tal modo, al priorizar sus problemas, es posible que confeccionen una larga y compleja lista de situaciones sociales y culturales, que habitualmente difiere de las prioridades listadas por autoridades y equipos de salud.

Distintos estudios han demostrado importantes divergencias de opinión entre proveedores de salud y usuarios. ${ }^{1,2}$ Esas divergencias se fundarían principalmente en los modelos conceptuales de salud y enfermedad que sostienen los sujetos como miembros de culturas definidas. El concepto de salud de los usuarios estaría inserto en un amplio contexto social y cultural. Salud es bienestar, y se relaciona con obtener mejor trabajo, tener alimentación adecuada, ser socialmente aceptado en la familia y en la comunidad, y actuar de acuerdo con las normas sociales y culturales del grupo. ${ }^{3}$ Los proveedores de salud, en cambio, verían la salud centrada en la enfermedad, en un contexto de facilidades tecnológicas y clínicas para abordar el proceso patológico, dejando muchas veces en un segundo plano las influencias sociales y culturales en el desarrollo y resolución de los problemas sanitarios. ${ }^{4}$

La atención primaria infantil constituye el primer nivel de acercamiento y la puerta de entrada de la población hacia el sistema de salud del Estado. En Chile y en particular en la región de la Araucania existe la red más extensa de consultorios y centros rurales primarios del país. Este sistema de atención se materializa a través de un programa gratuito de carácter nacional cuya meta es proteger y mejorar la salud de los niños menores de 15 años de edad. Dado el creci- miento de la red de atención primaria y la focalización de recursos hacia la población infantil en riesgo, la situación sanitaria global de los niños ha mejorado sostenidamente durante los últimos 20 años. Pero a pesar de los innumerables esfuerzos realizados para abordar la problemática sanitaria en la Araucania, ésta continúa siendo la zona de mayor vulnerabilidad infantil de la nación chilena. La presencia de los indicadores sanitarios y sociales más deteriorados del país lo demuestra. Por ejemplo: las tasas de mortalidad infantil, materna y perinatal por arriba del nivel nacional, el índice de escolaridad y de desarrollo humano más bajo del país, y las altas tasas de extrema pobreza, entre otros indicadores, construyen un perfil regional de riesgo para el crecimiento y desarrollo de su población infantil. A ello se suma que la región tiene $34.6 \%$ de población rural, y que más de $50 \%$ de ésta pertenece al grupo étnico más numeroso y pobre de Chile: el mapuche.

En el planteamiento de las nuevas políticas sanitarias se menciona que variables étnicas, culturales e históricas estarían condicionando la situación sanitaria de los niños en la Araucania. Sin embargo, existen escasos estudios que indaguen el peso de estas variables en la atención infantil.

Esta investigación se propone develar las dimensiones culturales en el proceso de atención primaria infantil desde la perspectiva de las madres mapuches y no mapuches que acuden a los centros de salud primaria en la región de la Araucania, en Chile.

\section{Material y metodos}

El presente trabajo es un estudio cualitativo basado en la teoría desarrollada por Strauss y Glaser, ${ }^{5}$ la cual plantea generar modelos teóricos a partir de un sistemático análisis de significados y conceptos que construyen los actores según su experiencia en el mundo cultural. Desde el punto de vista analítico, implica el desarrollo de tres etapas: identificación de temas emergentes, construcción de categorías conceptuales, y producción de modelos o cuerpos teóricos. ${ }^{6-9}$

El proceso de análisis de los datos textuales se realizó a través del programa Atlas.ti, ${ }^{*}$ y consistió en: 1) codificar las entrevistas transcritas para categorizar, separar y organizar el texto en unidades interpretativas menores; 2) construir familias de códigos a través de la comparación de sus significados; y 3) establecer redes de relación entre familias para descubrir los modelos teóricos que emergen de los datos.

* Muhr T. Atlas.ti: scientific software development. Berlín, 1997. 
Participaron en el estudio 94 madres inscritas en diferentes centros de atención primaria de la región de la Araucania. La selección se realizó mediante un muestreo por conveniencia, e incluyó madres con diferente adscripción étnica: mapuches $(60 \%)$ y no mapuches, de zonas rurales y urbanas, con y sin rol sociocultural en su comunidad $\mathrm{u}$ organizacióny y diferentes grados de escolaridad. El 42\% era bilingüe: Mapuche y Español. La edad promedio fue de 29 años, la escolaridad media de 8 años y la moda de hijos igual a 2 .

Los datos se recolectaron mediante entrevistas en profundidad que realizaron dos investigadores del proyecto en los domicilios de las madres, desde septiembre del 2003 hasta octubre del 2004. Se diseñó una guía de entrevista que trataba cuatro grandes temas: a) percepción de los problemas de salud infantil (identificación de sus causas, tratamientos y consecuencias); b) recursos de las madres para enfrentar las enfermedades de sus hijos; c) percepción de la atención de salud recibida en el centro de salud primario (trato, recursos físicos y humanos, acceso, pertinencia social y cultural); y d) sugerencias para adecuar la atención de salud a los usuarios. Cada entrevista duró aproximadamente una hora y media, se grabó previo consentimiento firmado de las madres, a quienes se les explicó el propósito del estudio y la metodología, lo que aseguró su participación voluntaria y anónima. El protocolo de investigación se sometió a la evaluación del comité de ética del Servicio de Salud Araucania, dependiente del Ministerio de Salud de Chile.

\section{Resultados}

El análisis de las 94 entrevistas evidenció tres dimensiones teórico-culturales involucradas en el proceso de atención primaria infantil: a) modelos etiológicos y explicativos de los problemas de salud infantil; b) el itinerario terapéutico refleja una complementación de saberes; c) competencia cultural del sistema de atención primaria infantil.

El cuadro I muestra una síntesis del análisis textual. Las dimensiones teóricas constituyen unidades culturales que ilustran las distintas esferas semánticas del estudio. Las categorías analíticas son los componentes conceptuales que otorgan significado a la dimensión teórica.

\section{Modelos etiológicos y explicativos de los problemas de salud infantil}

Del listado de problemas de salud infantil identificados por las madres, se obtuvo un sistema de clasificación general compuesto por los siguientes grupos: enfer-

\author{
Cuadro I \\ Dimensiones teóricas Y CATEgorías \\ ANALÍTICAS INVOLUCRADAS EN EL PROCESO \\ DE ATENCIÓN PRIMARIA INFANTIL. \\ región de la Araucania, Chile, 2003-2004 \\ Dimensiones teóricas \\ culturales \\ Categorias analíticas \\ I. Modelos etiológicos y \\ explicativos de los pro- \\ blemas de salud infantil. \\ I. Explicaciones culturales. \\ 2. Explicaciones ambientales. \\ 3. Explicaciones sociopolíticas. \\ II. Itinerario terapéutico \\ reflejo de una comple- \\ mentación de saberes \\ médicos. \\ I. La madre es el primer recurso tera- \\ péutico para sus hijos. \\ 2. El modelo etiológico de enfermedad \\ determina el curso terapéutico. \\ 3. Biomedicina y disponibilidad econó- \\ mica de la familia. \\ III. Competencia cultural \\ del sistema de atención \\ primaria infantil. \\ I. Barreras de lenguaje entre madres $y$ \\ personal de salud. \\ 2. Falta reconocimiento de la cultura sa- \\ nitaria de la familia. \\ 3. Diferentes expectativas de la relación \\ médico-paciente.
}

medades físicas o biológicas, alteraciones de la conducta social, y problemas del crecimiento y desarrollo del niño. En el primer grupo las madres incluyeron: tos, fiebre, vómitos, dolor, resfríos y neumonías, así como también enfermedades propias de la tradición popular e indígena: empacho, pasmo, mal de ojo y susto. La clasificación de una enfermedad como física o biológica no significa que las madres hayan aislado los componentes sociales, emocionales o psicológicos de la enfermedad, sino que la determinante principal por la cual todas dan cuenta del problema de salud es la condición física observable en el niño.

En el grupo de alteraciones de la conducta social, la mayoría de las madres identificó la "enfermedad de los nervios" como un problema de salud frecuente en la población infantil, especialmente en mayores de cinco años. Tales niños fueron caracterizados por: "irritarse con facilidad", "desobedecer órdenes de los padres o maestros", "pelear mucho con otros niños", y "agredirse a sí mismos".

Como problemas de crecimiento y desarrollo, la mayoría de las madres mencionó enfermedades de nacimiento o derivadas de problemas durante el parto: los niños "nacen enfermos" y reciben el nombre de "niños inocentes".

Todas las madres concibieron la enfermedad como una experiencia de sufrimiento: "tener un hijo enfermo es un sufrimiento muy grande" o "no hay peor dolor que tener un hijo enfermo". De acuerdo con ellas, 
el sufrimiento se basa en la incertidumbre, la ansiedad y el miedo frente al desenlace del problema, especialmente porque la infancia se consideró un periodo de fragilidad: "una nunca sabe qué va a pasar, si va a quedar enfermizo o se pone más grave", "es tal la desesperación que una no sabe qué hacer y dónde recurrir", "los niños son muy frágiles, su cuerpo es chiquito, no saben defenderse".

Basados en esta breve clasificación e identificación de problemas de salud, se analizaron los modelos explicativos e interpretativos de salud y enfermedad a los que recurren las madres. De este análisis surgieron tres tipos de explicaciones: ambientales, político-económicas y culturales.

\section{Explicaciones ambientales}

Las madres enfatizan las influencias que el medio ambiente tiene sobre la salud de los niños. Por ejemplo, el frío y la humedad fueron considerados como las principales causas de enfermedad infantil; al respecto, algunas dijeron: "los niños se pasan de frío", "la humedad les afecta los bronquios", "vivir en este hielo los enferma". Problemas tales como: "tos, fiebre, resfríos, gripe, neumonías, orinas sueltas, diarreas y pasmo" se explicaron como resultado de una combinación del frío y humedad. La mayoría de las madres mencionó la falta de recursos de las familias como un factor asociado a la enfermedad; por ejemplo: "mala calidad de las viviendas, falta de transporte escolar en zonas rurales, falta de vestuario apropiado y mala alimentación de los niños". Algunas mencionaron que la creciente contaminación ambiental, tanto en los sectores rurales como urbanos, es un factor que ha disparado las enfermedades en los niños: "las aguas están sucias y contaminadas por las industrias y basurales que se encuentran cada vez más cerca de las viviendas, eso de todas maneras hace que nuestros niños se enfermen". Todas las madres mapuches señalaron además que: "los niños se enferman más que antes porque la naturaleza está cambiando, los hombres están ensuciando la tierra, el agua y el aire".

\section{Explicaciones político-económicas}

Todas las madres plantearon que los problemas de salud de sus hijos se relacionan con su pobreza: "todo viene de un problema monetario, es esa la causa de todos nuestros problemas, la falta de oportunidad de los pobres". Dicen: "los niños pobres son más susceptibles de contraer cierta clase de enfermedades sólo por el hecho de vivir en la pobreza"; "el bajo ingreso económico de las familias impide comprar suficientes alimentos, medicamentos, o ir al centro de salud cuando es necesario"; y "la baja educación de los padres impide tener mejores trabajos y salarios". Así, la falta de recursos económicos y oportunidades de desarrollo de las familias son situaciones que colocan a los niños en mayor riesgo social y biológico. La perspectiva de estas madres es que la enfermedad surge como resultado de las condiciones de inequidad social. En este contexto, la mayoría planteó estar inmersa -como individuo- en fuerzas incontrolables que resultan en un ciclo de pobreza y enfermedad del cual es difícil salir sin políticas que superen las desigualdades sociales.

Los relatos de algunas madres campesinas ilustran la situación antes mencionada: "cuando los niños se enferman hay que vender alguna avecita para tener dinero para el bus porque no tenemos de donde sacar", "si el niño queda hospitalizado ahí se queda solito, no tenemos dinero para ir a la ciudad a visitarlo". En este mismo contexto una madre urbana dijo: "a veces tenemos que vender la leche que nos dan en el consultorio para comprar comida; los niños no se alimentan sólo de leche".

\section{Explicaciones culturales}

Un tercer grupo de explicaciones causales de enfermedad estuvo asociado a los modelos culturales de salud y enfermedad de las madres. Destacan: el modelo médico mapuche cuyas explicaciones se asocian a fenómenos naturales y mágico-religiosos; y el modelo médico popular, cuya explicación básica es el desbalance entre frío y calor. ${ }^{10}$ Por ejemplo, para madres mapuches y no mapuches, los resfríos y algunas gastroenteritis se explicaban porque "le entra frío en el cuerpo del niño"; en tanto que los problemas de salud mental se vincularon a causas sobrenaturales: "un mal realizado por alguien que le tiene envidia a la madre o a la familia", "castigo por no respetar las reglas de la comunidad" o "encuentros con seres sobrenaturales". Estas últimas explicaciones fueron mas frecuentes entre las madres mapuches y de origen rural. Asimismo, todas las madres mencionaron que el "mal de ojo", enfermedad bastante común entre los niños, era causada por: "personas que tienen poder o fuerza negativa que al admirar a un niño le provocan la enfermedad".

\section{El itinerario terapéutico refleja una complementación de saberes médicos}

La segunda dimensión teórica de este estudio fue el itinerario terapéutico que las madres recorren para solucionar los problemas de salud de sus hijos. De acuerdo con Kleinman y Psordas, ${ }^{11}$ el itinerario terapéutico consiste en la secuencia de actividades que los 
pacientes o sus familiares realizan para solucionar el problema de salud, y constituye un importante indicador de los recursos materiales, sociales y culturales de las familias.

El itinerario que las madres enunciaron refleja la multiplicidad de recursos terapéuticos utilizados para enfrentar la enfermedad de sus hijos. En este contexto, todos los itinerarios trazados demostraban una compleja combinación de recursos de medicina tradicional mapuche, medicina popular y biomedicina.

A partir de la indagación de los itinerarios surgieron tres categorías analíticas: 1) el primer recurso ante la enfermedad de los hijos es el conocimiento propio y el de parientes femeninos más cercanos, entre éstas alguna abuela del niño; 2) dependiendo del modelo etiológico de enfermedad, la madre busca ayuda de especialistas tradicionales o biomédicos; y 3 ) la selección del recurso terapéutico no depende sólo de los modelos etiológicos de enfermedad, sino también de los recursos económicos del núcleo familiar.

\section{La madre es el primer recurso terapéutico para sus hijos}

El conocimiento y experiencia propia fue el punto de partida en el itinerario terapéutico; de este modo la madre se convierte en la primera ayuda médica para el niño. Las entrevistadas mencionaron un copioso arsenal terapéutico transmitido a través de la familia, vecinas o medios de comunicación: hierbas medicinales, maniobras de primeros auxilios, medicamentos autoprescritos y comprados en farmacia, y ritos terapéuticos simples, entre otros. Si la madre desconoce la causa real de enfermedad o los tratamientos sintomáticos no logran el resultado deseado, se consulta a una mujer de la familia o vecina con conocimiento en enfermedades infantiles. La mayoría de las madres dijo no recurrir al centro de salud como primera instancia para resolver las enfermedades de sus hijos: lo hacen sólo si los niños continúan enfermos a pesar de los tratamientos realizados en el hogar.

\section{El modelo etiológico de la enfermedad determina el curso terapéutico}

Diferentes estudios etnográficos demuestran que la percepción de la causa de la enfermedad es un factor determinante en la búsqueda de ayuda terapéutica. ${ }^{12,13}$ En este estudio las madres señalaron que cuando la causa de la enfermedad son factores naturales: "aire, frío, calor" o proveniente de espíritus malignos: "mal, brujería, envidia", recurren a especialistas tradicionales de la cultura, quienes otorgan el tratamiento apropiado. Todas las enfermedades descritas por las madres tenían componentes mágico-religiosos y naturales. La identificación de los factores causales no fue sólo resorte para la acción de la madre, sino también de especialistas tradicionales; por ejemplo si el especialista manifiesta "no poder curar la enfermedad porque pertenece a la otra medicina", la madre busca ayuda en el sistema biomédico. Así, se teje una compleja trama de itinerarios, que además pueden ser secuenciales, paralelos o recursivos.

\section{Biomedicina y disponibilidad económica de la familia}

Especialmente para las madres campesinas uno de los factores más importantes en la decisión de llevar un hijo enfermo al médico es la disponibilidad de recursos económicos. Estas opinan que la atención médica es "difícil, costosa, sale mucho dinero". Desafortunadamente, los centros de atención primaria rural carecen de los insumos básicos en medicamentos, equipos y personal para proporcionar una atención oportuna y eficaz, motivo por el cual las madres campesinas deben viajar a los centros de salud urbanos. Ello implica gastos en transporte, alimentación y medicamentos, los cuales, la mayoría de las veces, sobrepasan las posibilidades económicas familiares. El tener dinero para solventar este tipo de gastos es también un factor determinante en la decisión sobre el curso terapéutico a seguir con los niños.

Las madres urbanas agregaron otro elemento. Plantearon que si bien los consultorios de atención primaria son gratuitos y accesibles, muchas veces éstos no tienen los medicamentos necesarios para cubrir su demanda y, por lo tanto, deben comprarlos en farmacias privadas. Por esta razón, muchas madres preferían acudir directamente al farmacéutico con el cual se evi$\tan$ la larga espera en los centros de salud: "no te dan jarabe, nada, que le des puro liquido, eso es todo, y a la casa. Si se mejoran, bueno, y si se agravan, al hospital", "mejor la farmacia, ahí pregunto, me ahorro tiempo y le compro remedios, pero sólo cuando puedo".

\section{Competencia cultural del sistema de atención primaria infantil}

La tercera dimensión teórica que emerge del estudio es la competencia cultural del sistema de salud para procurar una atención acorde con las características y necesidades de salud de la población infantil. Por competencia cultural se entiende un proceso de atención centrado en el paciente, y que otorga respuestas efectivas a las necesidades, valores y preferencias de los clientes (pacientes).$^{14} \mathrm{Al}$ respecto, la mayoría de las madres planteó que el sistema carece de adecuación 
cultural para resolver los problemas de salud de los niños. Las categorías analíticas involucradas en este análisis apuntan a tres elementos: a) barreras de lenguaje entre madres y personal de salud, b) falta de reconocimiento de la cultura sanitaria de la familia y c) diferentes expectativas de la relación medico-paciente.

\section{Barreras de lenguaje}

Todas las madres mencionaron importantes barreras de lenguaje con el personal de salud debido a dos razones fundamentales. Primero, no comprenden el lenguaje técnico biomédico: "las indicaciones de los médicos son incomprensibles", "si uno les pregunta qué significa lo que dicen, los médicos a veces se enojan, así que mejor no preguntar". Segundo, en el caso de la población mapuche que tiene además su propio idioma, las barreras de lenguaje son a la vez idiomáticas; una de estas madres dijo: "te dicen tiene el virus no se cuanto, y uno ni siquiera le comprende porque ellos no son mapuche, si fueran, ahí sí uno le podría entender".

\section{Falta de reconocimiento de la cultura sanitaria de la familia}

Para las madres el conjugar las explicaciones médicas con sus propios modelos de enfermedad resulta un proceso muy controversial. Muchas de ellas plantearon que ocultan al personal de salud sus ideas sobre la enfermedad de sus hijos, y a veces no siguen el tratamiento médico porque consideran que algunos medicamentos son inapropiados para la enfermedad. Estas actitudes se basan en la percepción de que el personal de salud no respeta sus creencias y valores culturales: "el médico no toma en cuenta la opinión de una", "se molestan y la retan si uno le dice que piensa", "a veces los remedios son malos, le hacen más mal, yo no se los doy (....) ¿qué saco con decirle si se enoja?".

\section{Expectativas de la relación médico paciente}

$\mathrm{Al}$ respecto, la mayoría de las madres mencionó que al acudir al centro de salud sus expectativas son "conversar sobre la enfermedad", "comprender más profundamente los problemas", y que el médico o la enfermera "examinen completamente al niño, no sólo pregunten por qué lo llevo y vean esa parte". Sin embargo, la mayoría de las madres señaló que: "los médicos no explican ni preguntan acerca del cuidado de los niños", "no tienen tiempo para examinarlos bien", "no son comprensivos con lo que uno necesita". En general, calificaron la relación médico-paciente como "fría e impersonal" y "no centrada en lo que uno realmente necesita".

\section{Discusión}

El hallazgo más relevante del estudio ha sido la identificación de las tres dimensiones culturales que componen la problemática de atención primaria infantil en la región de la Araucania: los modelos explicativos de salud-enfermedad, el itinerario terapéutico y la competencia cultural del sistema de atención.

En los discursos de las madres ha sido posible identificar que las explicaciones culturales, ambientales y sociopolíticas determinan el análisis de los problemas de salud infantil. Estas explicaciones se tejen en una compleja trama de causalidades, entre las que predominan los modelos mágico-religioso y popular de salud. La breve taxonomía descrita por las madres demuestra la integralidad del concepto de salud, percibido como el estado de bienestar general y desarrollo armónico de las potencialidades del niño, al mismo tiempo que la enfermedad se concibe como un estado de sufrimiento e incertidumbre.

En relación con el itinerario terapéutico trazado por las madres, es posible observar la articulación de recursos provenientes de los sistemas de salud tradicional-indígena, popular y biomédico. A la par, se destaca que el conocimiento y sabiduría de las madres y mujeres de la familia constituye la primera fuente de ayuda terapéutica para los niños enfermos. Es también notable que para la mayoría de las madres el acudir al sistema biomédico no constituye la primera elección frente a la enfermedad de sus hijos. En esta decisión gravitan dos factores fundamentales: la percepción de la etiología de la enfermedad y la disponibilidad de recursos económicos de la familia para enfrentar los gastos derivados de la atención médica.

La tercera dimensión cultural resultante del análisis fue la competencia cultural del sistema de atención primaria infantil. En este aspecto todas las madres señalaron que la atención de salud no es culturalmente pertinente a sus necesidades y expectativas y en este sentido subrayaron la ausencia de un proceso comunicativo en la relación médico-paciente, que se funda en factores como: barreras de lenguaje, falta de conocimiento del personal de salud sobre la cultura sanitaria de las madres, y escaso diálogo y comunicación durante la atención de salud.

Los resultados de este estudio corroboran postulados teóricos de la antropología médica que sostienen que la brecha entre equipos de salud y pacientes ocurre, entre otras razones, por los modelos etiológicos culturales de salud y enfermedad, así como por la pertenencia a diferentes estratos socio-educacionales. Las diferencias culturales podrían abordarse median- 
te el conocimiento de la cultura del paciente, pero también generando un proceso de comunicación cultural que contemple: escuchar con atención, explicar, reconocer el modelo cultural del paciente, recomendar más que indicar, y negociar explicaciones y tratamientos terapéuticos. ${ }^{15}$ Estrategias de este tipo y antecedentes como los que se presentan en este estudio parecen poco conocidos en el ámbito de la salud publica y la epidemiología; sin embargo, tienen gran importancia para diseñar políticas y modelos de atención intercultural en salud que logren dar satisfacción a las necesidades y expectativas de las madres en áreas de gran diversidad étnica y cultural como la región de la Araucania en Chile. Al mismo tiempo, investigaciones futuras deberían abordar el tema de las brechas culturales y sociales que emergen de la interacción paciente y personal de salud.

\section{Referencias}

I. Kleinman A. Patients and healers in the context of culture. Berkeley: University Press, 1980.

2. Helman C. Culture, health, and illness. Oxford: ButterworthHeinemann, 1994.

3. Hanhn R. Sickness and healing: An anthropological perspective. New Haven:Yale University Press, 1995.
4. Bryant J. Health and developing world. Cornell: University Press, 1975. 5. Strauss A, Glaser B. Discovery of substantive theory:A basic strategy underlying qualitative research. Chicago: Markham, 1970.

6. Bernard R, Ryan G. Text análisis: Qualitative and quantitative methods. En: Russel B, comp. Handbook of methods in cultural anthropology. Walnut Creek: Altamira Press, 1998: 65-87.

7. Potter J. Discourse analysis as a way of analyzing naturally occurring talks. En: Silverman D, comp. Qualitative research:Theory, method and practice. Thousand Oaks: Sage Publications, 1997: 97-II2.

8. Miles M, Huberman A. Qualitative data analysis. Newbury Park: Sage Publications, 1994.

9. Prior L. Text and context in qualitative research. En: Silverman D, comp. Qualitative research:Theory, method and practice. Thousand Oaks: Sage Publications, 1997: I 3-145.

10. Harwood A. The hot-cold theory of disease: implications for the treatment of Puerto Rican patients. En: Brown P, comp. Understanding and applying medical anthropology. Mountain View: Mayfield Publishing Company, 1998: 25I-260.

II. Kleinman A, Csordas T. The therapeutic process. En: Sargent C, Johnson T, comp. Medical anthropology: contemporary theory and method. Westport: Praeger Publishers, 1996:3-21.

12. Nichter M. Anthropological approaches to the study of ethnomedicine. Philadelphia: Gordon and Breach Science Publishers, 1992.

13. Janzen J.Therapy management: concept, reality, process. Med Anthropol Q 1987; I:68-84.

14. Betancourt J. Cultural competence: marginal or mainstream movement?. N Engl J Med 2004; 10:953-955.

15. Berlin E, Fowkes W.A teaching framework for cross cultural health. En: Brown P, comp. Understanding and applying medical anthropology. Mountain View: Mayfield Publishing Company, 1998: 303-309. 